\title{
Fluidity and supercriticality of the QCD matter created in relativistic heavy ion collisions
}

\author{
Jinfeng Liad* and Volker Koch \\ Nuclear Science Division, Lawrence Berkeley National Laboratory, \\ MS70R0319, 1 Cyclotron Road, Berkeley, CA 94720.
}

\begin{abstract}
In this paper we discuss the fluidity of the hot and dense QCD matter created in ultrarelativistic heavy ion collisions in comparison with various other fluids, and in particular suggest its possible supercriticality. After examining the proper way to compare non-relativistic and relativistic fluids from both thermodynamic and hydrodynamic perspectives, we propose a new fluidity measure which shows certain universality for a remarkable diversity of critical fluids. We then demonstrate that a fluid in its supercritical regime has its fluidity considerably enhanced. This may suggest a possible relationship between the seemingly good fluidity of the QCD matter produced in heavy ion collisions at center of mass energy of $\sqrt{s}=200 \mathrm{AGeV}$ and the supercriticality of this matter with respect to the Critical-End-Point on the QCD phase diagram. Based on such observation, we predict an even better fluidity of the matter to be created in heavy ion collisions at LHC energy and the loss of good fluidity at certain lower beam energy. Finally based on our criteria, we analyze the suitability of a hydrodynamic description for the fireball evolution in heavy ion collisions at various energies.
\end{abstract}

PACS numbers: 12.38.Mh, 25.75.-q, 47.75.+f

\section{INTRODUCTION}

The exploration of the QCD phase diagram as well as the quantitative characterization of QCD matter is one of the most interesting challenges and questions in strong interaction physics. Hot and dense QCD matter can be created in the laboratory by means of heavy ion collisions, and experiments at the CERN Super Proton Synchrotron (SPS) and at the Relativistic Heavy Ion Collider (RHIC) have, over the years, revealed many intriguing and unexpected properties of this matter. For example the measurement of an unexpectedly large elliptic anisotropy $v_{2}$ 1] can be reproduced within the framework of hydrodynamics [2], at least for low transverse momenta $\left(p_{t}\right)$. This observation has led to the conjecture, that the matter produced in these collisions is strongly interacting, with nearly ideal fluidity [3]. Several microscopic explanations for this behavior have been suggested [4] [5] [6], for example the "magnetic scenario" which features the coexisting electric and magnetic components of the plasma with the magnetic one ultimately enforcing the QCD confinement transition.

Arguments for the nearly ideal fluidity at center of mass energies of $\sqrt{s}=200 \mathrm{AGeV}$, quantitatively represented by a rather small ratio of shear-viscosity over entropy-density, $\eta / s$, have come from different directions. Firstly, the $v_{2}$ data measured at RHIC have provided rather stringent constraints on viscous hydrodynamic calculations: the current status based on this approach is that an upper limit $\eta / s \leq 6 /(4 \pi)$ may be set [2]. (Some caveats on interpretation of $v_{2}$ data however should be

\footnotetext{
*Electronic address: jliao@lbl.gov
}

$\dagger$ Electronic address: vkoch@lbl.gov kept in mind, such as hidden non-collective contributions to $v_{2}$ [7] or alternative mechanism of producing $v_{2}[8]$ ). Secondly, recent developments from the AdS/CFT correspondence [9] have hinted at a universal lower bound $\eta / s \geq 1 /(4 \pi)$. While in principle violating examples have been found (see detailed discussions in e.g. [10]), for all practical purposes this value serves as a useful benchmark for good fluidity of relativistic fluids. Finally, the fluidity of the QCD fluid has been compared [11, 12 to a few commonly known substances like Helium-4, water, nitrogen etc, using the measure $\eta / s$ : it has been concluded that the QCD fluid has smaller $\eta / s$ as compared with all those normal substances.

In this paper we will revisit the concept of fluidity. For a sensible discussion of fluidity one needs to clearly distinguish the concept of fluidity from the concept of applicability and validity of hydrodynamics. The concept of fluidity of a substance can only be meaningful if the fluidity is defined exclusively in terms of properties of the substance itself. The question of applicability of hydrodynamics, on the other hand, requires the effective mean free path ${ }^{1}$ to be small compared to the size of the system or rather the typical wavelengths of the excitations which are supposed to be described within hydrodynamics. Consequently, in this case we compare a property of the system, the mean free path, with an external scale characterizing the situation we want to describe within

\footnotetext{
1 To simplify the argument, in the rest of the introduction we will use the familiar concepts of "mean free path" and particles, noting that these are limited to a kinetic description of a sufficiently dilute system. In section [II we will derive a more general expression for the relevant length scale which will not require the applicability of kinetic theory. In the kinetic limit it reduces to the mean free path.
} 
hydrodynamics, for example a sound mode of a given wavelength. And given a sufficiently large external scale, hydrodynamics is always applicable. For example we observe sound propagation in both water and air, and yet one would be inclined to assign a better fluidity to water than to air. Therefore a meaningful definition of $f l u$ idity needs to measure the effective mean free path in terms of a length scale inherent to the substance itself. One obvious choice is the interparticle distance, or more generally, the density-density correlation length. This allows the comparison of substances with vastly different inherent length scales, such as water vs. a Quark-Gluon Plasma. Obviously, the minimum wavelength (in meters) for sound propagation even in a weakly interacting QGP is still considerably shorter than that of a strongly interacting atomic or molecular substance. However, if we ask the question "what is the minimal wavelength for sound propagation measured in units of the interparticle distance?" then a comparison between these substances becomes meaningful, and the strongly interacting substance will likely exhibit a better fluidity.

Following these general considerations, in this paper we discuss the fluidity of the hot and dense QCD matter created at RHIC by comparing it with normal nonrelativistic fluids and by applying valuable insights from those fluids. While such a comparison is not new (see e.g. 11] [12]), we differ from all previous approaches in a few distinct points. First of all, we carefully examine the difference between the relativistic and non-relativistic fluids. This includes their different inertia: the relativistic inertia, i.e. the enthalpy, reduces to the mass density in the non-relativistic regime. This also includes the choice of reference scale, as discussed above: while the temperature may serve as a reasonable estimator for the interparticle distance for relativistic fluids, it certainly does not in the non-relativistic limit. Based on these considerations, we propose a new measure of fluidity for comparing various fluids. Furthermore we emphasize, for the first time, the possible relevance of the so-called supercritical fluid for the matter produced at RHIC and discuss important implications for the expected fluidity for the matter produced in heavy ion collisions at different center of mass energies. We will also elaborate on potential consequences for the search of the QCD Critical-End-Point via a beam energy scan.

As just discussed, a closely related, but different, question is the applicability of hydrodynamics in heavy ion collisions at various beam energy $\sqrt{s}$. For the description of the dynamical evolution of system within hydrodynamics to be valid the length scale characterizing the variations of the system needs to be large compared to the effective mean free path of the (quasi) particles within the fluid [2] 15]. Thus, good fluidity of the underlying matter may not guarantee applicability of hydrodynamics, if the typical gradients of the flow field are large, e.g. when the system size is very small. Based on an analysis of sound wave attenuation, we will provide quantitative criteria for the applicability of hydrodynamics, and evaluate the situations at SPS, RHIC, and LHC respectively.

This paper is organized as follows: In Section [I we will discuss the difference and relation between a nonrelativistic(NR) fluid and a relativistic(R) fluid. In Section [II] we will then propose a new fluidity measure, which is applicable for both relativistic and nonrelativistic systems. Based on this new measure we will compare various fluid systems and demonstrate the improvement of fluidity in a fluid's supercritical regime. In Section [V] we will use Lattice QCD results to construct equal-pressure lines on the QCD phase diagram. We then discuss the relationship between fluidity and supercriticality for heavy ion collisions, the evolution of fluidity with beam energies, and its implications for the search of the QCD Critical-End-Point(CEP). Finally in Section $\nabla$ the applicability of hydrodynamics in heavy ion collisions at various energies will be discussed.

\section{RELATIVISTIC AND NON-RELATIVISTIC FLUIDS}

When comparing a relativistic $(\mathrm{R})$ with a nonrelativistic (NR) fluid, one needs to carefully keep track of the mass terms, which customarily are neglected in nonrelativistic thermodynamics. In non-relativistic thermodynamics, the basic thermodynamic relation

$$
E_{N R}=T S-p V+\mu_{N R} N
$$

does not take into account the mass of the particles. Here $E_{N R}$ refers to the kinetic and interaction energy of the particles. Similarly, the chemical potential, which represents the increase of energy by the addition of one extra particle, does not account for the particle's mass. The relativistic version of the basic thermodynamic relation,

$$
E_{R}=T S-p V+\mu_{R} N,
$$

on the other hand, takes into account the particle masses. Its non-relativistic limit can be obtained by simply including the mass terms in both the energy and the chemical potential

$$
\begin{aligned}
& E_{R}=E_{N R}+m \\
& \mu_{R}=\mu_{N R}+m .
\end{aligned}
$$

As we will discuss below (see also [17, 18]), the thermodynamic quantity entering hydrodynamics is the enthalpy density, $w$ defined as

$$
w_{R}=\epsilon+p=T s+\mu_{R} n
$$

where $\epsilon$ is the energy density, $p$ the pressure, $s$ the entropy-density, and $n$ is the particle-density. As we will show, the non-relativistic limit of hydrodynamics involves the non-relativistic limit of the enthalpy, $w_{R}$, including the mass term

$$
w=T s+\left(\mu_{N R}+m\right) n \stackrel{T \ll m}{\longrightarrow} m n \equiv \rho
$$


and it is dominated by the mass density. In the ultrarelativistic limit $T \gg \mu_{R}$, on the other hand, the enthalpy density is given by

$$
w^{T \gg \mu_{R}} T s .
$$

The kinematic viscosity, which is defined as the ratio of the shear viscosity over the enthalpy density

$$
\nu=\frac{\eta}{w}
$$

usually serves as a measure for dissipation 17]. While the widely used ratio of shear-viscosity over entropy density, $\eta / s$ is indeed related with the kinematic viscosity for ultra-relativistic systems, it misses the dominant mass term for a non-relativistic fluid.

In the following, we further demonstrate this point both in thermodynamics and in hydrodynamics ${ }^{2}$.

\section{A. Thermodynamics}

We start with the example of a classical (Boltzmann), relativistic free gas at temperature $T$ and fixed (net)particle density $n=n_{P}-n_{\bar{P}}$. Following standard statistical mechanics in e.g. [16], the Helmholtz free energy density is given by

$$
\begin{aligned}
f(T, n)= & n\left(k_{B} T\right)\left\{-\sqrt{1+\left(\frac{2 I\left[\beta m c^{2}\right]}{n \tilde{\lambda}^{3}}\right)^{2}}\right. \\
& \left.+\ln \left[\frac{\frac{n \tilde{\lambda}^{3}}{\left[\beta m c^{2}\right]}+\sqrt{\left(\frac{n \tilde{\lambda}^{3}}{I\left[\beta m c^{2}\right]}\right)^{2}+4}}{2}\right]\right\}
\end{aligned}
$$

with $\beta \equiv 1 /\left(k_{B} T\right)$ and $\tilde{\lambda}=\left(2 \pi^{2}\right)^{1 / 3}(\beta \hbar c)$. Here we have introduced the re-scaled momentum integral, $\tilde{p}=\beta p c$,

$$
I\left[\beta m c^{2}\right] \equiv \int_{0}^{\infty} d \tilde{p} \tilde{p}^{2} e^{-\sqrt{\tilde{p}^{2}+\left(\beta m c^{2}\right)^{2}}} .
$$

Using the usual thermodynamic relations we obtain expressions for other quantities, such as the chemical potential and the energy density:

$$
\begin{aligned}
\mu= & \frac{\partial f}{\partial n}=\left(k_{B} T\right) \ln \left[\frac{\frac{n \tilde{\lambda}^{3}}{I\left[\beta m c^{2}\right]}+\sqrt{\left(\frac{n \tilde{\lambda}^{3}}{I\left[\beta m c^{2}\right]}\right)^{2}+4}}{2}\right] \\
\epsilon & =f-T \frac{\partial f}{\partial T} \\
& =n\left(k_{B} T\right) \sqrt{1+\left(\frac{2 I}{n \tilde{\lambda}^{3}}\right)^{2}}\left(3-\left(\beta m c^{2}\right) \cdot \frac{I^{\prime}}{I}\right)
\end{aligned}
$$

2 To better keep track of the mass terms, in the following two subsections we make explicit the dependence on the speed of light $c$, the Boltzmann constant $k_{B}$ and the Planck constant $\hbar$, while in the rest of the paper we use natural units with these constants taken to be unity.
Next we examine the non-relativistic limit by taking $\beta m c^{2} \rightarrow \infty$ in function $I[y], \mathrm{Eq} 10$, (and its derivative $\left.I^{\prime} \equiv d I[y] / d y\right)$ :

$$
\begin{aligned}
& f \rightarrow N R: n m c^{2}+n\left(k_{B} T\right)\left[\ln \left(n \lambda^{3}\right)-1\right] \\
& \mu \rightarrow N R: m c^{2}+\left(k_{B} T\right) \ln \left(n \lambda^{3}\right) \\
& \epsilon \rightarrow N R: n m c^{2}+\frac{3}{2} n\left(k_{B} T\right)
\end{aligned}
$$

with $\lambda \equiv\left(2 \pi \hbar^{2} / m k_{B} T\right)^{1 / 2}$. Obviously, in the nonrelativistic regime the energy associated with the rest mass dominates the chemical potential, the free energy density, the energy density, and the enthalpy density. Contrary to that, neither entropy density $s=(\epsilon-f) / T$ nor the pressure $p=\mu n-f$, have an explicit dependence of the mass term, as intuitively expected.

\section{B. Hydrodynamics}

We now turn to the hydrodynamics in the relativistic and non-relativistic regime. Since the non-relativistic limit of relativistic hydrodynamics is discussed in textbooks [17, 18], we will be brief here and just remind ourselves of the essential points, in particular how the relativistic inertia i.e. the enthalpy density $w$ is replaced in the non-relativistic limit by the mass density $\rho c^{2}$. We start with the non-relativistic Navier-Stokes (N-S) equation $^{3}$ :

$$
\left[\partial_{t}+\vec{v} \cdot \vec{\nabla}\right] \vec{v}=-\frac{\vec{\nabla} p}{\rho}+\frac{\eta}{\rho} \nabla_{j} \vec{\Sigma}^{j i}
$$

with the non-relativistic shear tensor $\Sigma^{j i}=\partial_{j} v_{i}+\partial_{i} v_{j}-$ $\frac{2}{3} \delta_{j i} \vec{\nabla} \cdot \vec{v}$. The corresponding relativistic N-S equation is given by ${ }^{4}$ :

$$
\begin{aligned}
\gamma^{2}\left[\partial_{t}+\vec{v} \cdot \vec{\nabla}\right] \vec{v}=\quad & -\frac{1}{w / c^{2}}\left[\vec{\nabla} p+\frac{\vec{v}}{c} \partial_{0} p\right] \\
& +\frac{\eta}{w / c^{2}} \partial_{\nu} \vec{\Sigma}^{\nu i}
\end{aligned}
$$

with the relativistic shear tensor $\Sigma_{\mu \nu}=c\left[\partial_{\mu} u_{\nu}+\partial_{\nu} u_{\mu}-\right.$ $\left.(u \cdot \partial) u_{\mu} u_{\nu}+\frac{2}{3}\left(u_{\mu} u_{\nu}-g_{\mu \nu}\right)(\partial \cdot u)\right], \gamma=1 / \sqrt{1-v^{2} / c^{2}}$, $u_{\mu}=\gamma(1, \vec{v} / c)$, and $\partial_{0}=\frac{1}{c} \partial_{t}$. In the non-relativistic limit one has $\gamma \rightarrow 1$, and $w / c^{2} \rightarrow \rho$ and thus in leading order of $v / c$ recovers the non-relativistic Navier-Stokes equation [17].

To further elaborate the point, let us consider the propagation and attenuation of a sound wave of frequency $\omega$

${ }^{3}$ In this discussion of N-S equation we neglect bulk viscosity and assume constant shear viscosity across the fluid.

${ }^{4}$ It is well-known that for relativistic hydrodynamics the derivative expansion to only first order, i.e. the N-S form, has causality problem and a consistent treatment requires higher orders in derivative expansion, see e.g. [2]. 
and wave vector $k=2 \pi / \lambda_{s}$ ( $\lambda_{s}$ is the wavelength ) in the presence of dissipation, characterized by the shear viscosity $\eta$. The dispersion relation for the wave is given by

$$
\omega=c_{s} k-\frac{i}{2} k^{2} \times\left\{\begin{array}{lr}
\frac{\frac{4}{3} \eta}{w / c^{2}}, & \text { R fluid } \\
\frac{4}{3} \eta & \text { NR fluid }
\end{array}\right\}
$$

To take into account possible bulk viscosity $\zeta$, one simply makes the replacement $\frac{4}{3} \eta \rightarrow \frac{4}{3} \eta+\zeta$. Furthermore the speed of sound $c_{s}$ is given by

$$
c_{s}=\left\{\begin{array}{ll}
\sqrt{\frac{\partial P}{\partial\left(\epsilon / c^{2}\right)}}, & \text { R fluid } \\
\sqrt{\frac{\partial P}{\partial \rho}}, & \text { NR fluid }
\end{array}\right\}
$$

As one can see, the same correspondence $(w, \epsilon)_{R} \rightarrow$ $\left(\rho c^{2}\right)_{N R}$ appears again as in the thermodynamics Eq.(13).

\section{Discussion on $\eta / s$}

We end this section by a discussion of the ratio of shearviscosity over entropy-density-ratio, $\eta / s$, which has attracted a considerable interest in various fields of physics.

We first recall, from the perspective motivated by studying the QGP via heavy ion collisions, why $\eta / s$ is a useful measure for the relativistic fluid, as was first pointed out in the seminal paper [19]. Consider a Bjorken-type longitudinal expansion of the quark-gluon plasma (with low baryonic density) formed in heavy ion collisions: its system size is limited by $c \tau$ at early time. With the presence of shear viscosity $\eta$, there will be dissipative effect (e.g. entropy generation) characterized by the ratio $\frac{\eta / w}{\tau}$ (roughly the Knudsen number) which, by thermodynamic relation Eq.(77) $w \simeq T s$, leads to $\frac{\eta}{s} \frac{1}{T \tau}$. To ensure a controllable dissipative correction to the ideal hydrodynamics, one requires firstly $\eta / s$ is small enough and, secondly, $T \tau$ is of the order 1 or bigger. The former shall be a property of the underlying QGP while the latter means the viscous effect is most severe at early time and the hydrodynamic evolution may not be a good approximation at too early time. (Note in this discussion we adopted natural relativistic units with $c=1$ ). One can draw two conclusions from this discussion: (a) $\eta / s$ can serve as a good measure of fluidity for a relativistic fluid and the smaller it is the better the fluidity; (b) the ability of $\eta / s$ to serve such a role is actually inherited from $\eta / w$.

The discussion above, however, leads to the observation that $\eta / s$ for a non-relativistic fluid does NOT necessarily provide a good measure for its fluidity, because the role of the relativistic $\eta / w$ corresponds to the nonrelativistic $\eta / \rho$. This is certainly not a new lesson: it has been known from Navier and Stokes's time that what really matters for usual non-relativistic fluids' fluidity is not the dynamical viscosity $\eta$ itself but rather the socalled kinematic viscosity $\eta / \rho$ (see e.g. [17]). Indeed by looking at actual data for water in the liquid and vapor phase at the same pressure one finds that liquid water has about one order of magnitude bigger shear viscosity, $\eta$, but nonetheless "wins the fluidity contest" since its kinematic viscosity, $\eta / \rho$, is about two orders of magnitude smaller than that of the vapor phase. We note that, the expression $\eta / w$ for a relativistic fluid is a relativistic version of the kinematic viscosity (see also related discussions in [3]). To conclude, we emphasize that for a non-relativistic fluid $\eta / \rho$ is different from $\eta / s$ (as is evident from the thermodynamics discussion) and only $\eta / \rho$ serves as a good measure of fluidity (as is evident from the hydrodynamics discussion). This also implies that using $\eta / s$ to compare the fluidity between a relativistic fluid (like the QGP, the AdS/CFT plasma) and a nonrelativistic fluid (like Helium, water or cold Fermion gas [20] ) may not be as informative as one would expect.

\section{A NEW FLUIDITY MEASURE}

In this section we propose a new fluidity measure that is suitable for comparison between relativistic and nonrelativistic fluids. In the following we will analyze the propagation of sound modes in the presence of dissipation (viscosity) and ask ourselves under what condition the dissipative (viscous) terms in the equations prevent sound from propagating. The advantage of this strategy is that we stay entirely within the framework of viscous hydrodynamics and do not need to make additional assumptions, such as the applicability of kinetic theory. As a consequence our fluidity measure will be expressed in terms of well defined quantities such as the shear viscosity, the speed of sound, etc.

We start with the sound dispersion relation in Eq.(16). By requiring the imaginary part of the frequency, $\mathcal{I} m \omega$, to be small in magnitude as compared with its real part Rew, we obtain:

$$
\begin{aligned}
\left|\frac{\mathcal{I} m \omega}{\mathcal{R} e \omega}\right|<<1 & \rightarrow \\
\lambda_{s} & =\frac{2 \pi}{k}>>\frac{4 \pi}{3} L_{\eta} \\
L_{\eta} & \equiv\left\{\begin{array}{cc}
\frac{\eta}{\left(w / c^{2}\right) c_{s}}, & \text { R fluid } \\
\frac{\eta}{\rho c_{s}}, & \text { NR fluid }
\end{array}\right\}
\end{aligned}
$$

The above equation implies that if a sound wave has its wavelength $\lambda_{s}$ comparable (or even smaller) than $L_{\eta}$, it will be quickly damped on (or shorter than) a time scale of its period and spatially on a length scale about (or shorter than) its wavelength, which essentially means it can not propagate away in the medium. Therefore, the physical meaning of the length $L_{\eta}$ introduced above is to provide a measure for the minimal wavelength of a 
sound wave to propagate in such a viscous fluid ${ }^{5}$. A more quantitative criteria will be discussed in Section $\mathrm{V}$ and given in Eq.(27).

Furthermore the length $L_{\eta}$ has the meaning of an effective mean-free-path (MFP) in terms of microscopic fluid particle motion. This becomes transparent in a weakly coupled gas: taking the non-relativistic gas as an example, the shear viscosity according to kinetic transport is

$$
\eta \sim \rho v_{T} l_{M F P}
$$

while the speed of sound is

$$
c_{s}=\sqrt{\frac{\partial P}{\partial \rho}} \sim \sqrt{\frac{k_{B} T}{M}} \sim v_{T}
$$

with $v_{T}$ the thermal velocity. These lead to the combination

$$
L_{\eta}=\frac{\eta}{\rho c_{s}} \sim l_{M F P}
$$

Unlike the mean-free-path which is conceptually intuitive but practically not easily computable or measurable (e.g. for fluids), the length $L_{\eta}$ is well-defined by macroscopic properties of the fluid and thus of practical use.

We emphasize that involving the speed of sound in the definition of length scale $L_{\eta}$ is essential. This can be seen already from a dimensional argument: $\eta / \rho$ or $\eta /\left(w / c^{2}\right)$ has the dimension of $[\text { Length }]^{2}[\text { Time }]^{-1}$. To turn this into a length scale one needs to divide it by a quantity of the dimension [Length] [Time $]^{-1}$ i.e. a velocity. The natural choice here is the speed of sound as a characteristic of the macroscopic matter. The speed of light, $c$, on the other hand is not suitable here since it is neither a property of any specific substance nor should it be of any relevance to non-relativistic fluids, such as water. Since the speed of sound changes considerably close to a phase-transition or a rapid crossover, its inclusion in the fluidity measure $\mathcal{F}$ and in the effective mean free path $L_{\eta}$ is essential.

Next we need to introduce another meaningful length scale to make a dimensionless ratio: this becomes a necessity when comparing fluids at vastly different scales. In case of the well known dimensionless ratios like the Knudsen and Reynolds numbers, an external length scale characteristic of the fluid motion is introduced, like the

\footnotetext{
5 We note that other types of dissipative processes like thermal conduction may also be present and thus introduce different length scales. For example when the sound wave period $\tau_{s} \sim 1 /\left(c_{s} k\right)$ becomes larger than the thermal relaxation time scale $\tau_{T} \sim 1 /\left(D_{T} k^{2}\right)$ (with $D_{T}$ the thermal diffusivity) at wavelength smaller than $D_{T} / c_{s}$, then heat transport becomes rather efficient and the sound propagation becomes isothermal (see examples in e.g. [21]). Nevertheless additional sources for dissipation do not change the fact that the "good" sound modes shall have their wavelengths (at least) larger that the $L_{\eta}$ set by shear viscosity only.
}

diameter of a pipe or the size of a moving object inside the fluid, etc. In the context of relativistic fluids created in heavy ion collisions, there have been scaling studies of collective flow for lower energy collisions based on the Reynolds number in [13] and more recently for higher energy collisions based on the Knudsen number in 14] (see also related discussions in [15]). The external length scale in those numbers, however, is not an intrinsic property of the fluid that we would like to invoke for comparing fluids across vastly different scales. Instead, a de-correlation length of certain density-density spatial correlator gives a natural scale of short-range order in the system. In most cases this de-correlation length is simply set by the inter-(quasi-)particle distance. For a non-relativistic fluid "particles" and their number density $n$ are well-defined, and thus the inter-particle distance is also a well-defined length scale:

$$
L_{n} \equiv \frac{1}{n^{\frac{1}{3}}}
$$

For relativistic fluid it is less straightforward. For example a QGP with $\mu_{B}=0$ and thus $n_{B}=0$ can still have substantial numbers of quarks and gluons. For such a relativistic fluid a simple estimate can be made via the entropy density, i.e. $n \sim \frac{s}{4 k_{B}}$. Another way is to calculate the de-correlation length of e.g. the correlator $<T_{00}(\vec{x}, 0) T_{00}(\overrightarrow{0}, 0)>$ as has been done in recent lattice work 22] which finds a short range order about $0.6 / T$ for QGP in $1-2 T c$, in reasonable agreement with estimate from $n \sim \frac{s}{4 k_{B}}$. There could still be academic examples where the entropy density can be infinite while the short-range order does not vanish: AdS/CFT offers such an example in which the entropy density goes as $s \propto N_{c}^{2} T^{3} \rightarrow \infty$ (in the large $N_{c}$ limit) implying $1 / n^{1 / 3} \rightarrow 0$ while spatial correlators like $<T_{\mu \nu}(\vec{x}, 0) T_{\mu \nu}(\overrightarrow{0}, 0)>$ give a non-zero de-correlation length $\sim 1 / T$. We will return to these issues in the Subsection IIC.

Finally by taking a ratio of the two length scales, we arrive at a fluidity measure

$$
\mathcal{F} \equiv \frac{L_{\eta}}{L_{n}}
$$

Below we will first show that the measure works well for non-relativistic fluids and bears certain universality for critical fluids. We will then show that the so-called supercritical fluids have even better fluidity. At the end we present a comparison of various interesting fluids. Following [11, 12], we have extensively used the measured data for various fluids from the NIST WebBook [23].

\section{A. Critical Fluids}

We first examine various fluids at fixed critical pressure $P=P_{c}$. In Fig (left), the proposed fluidity measure $\mathcal{F}=L_{\eta} / L_{n}$ is plotted for fifteen different substances at 

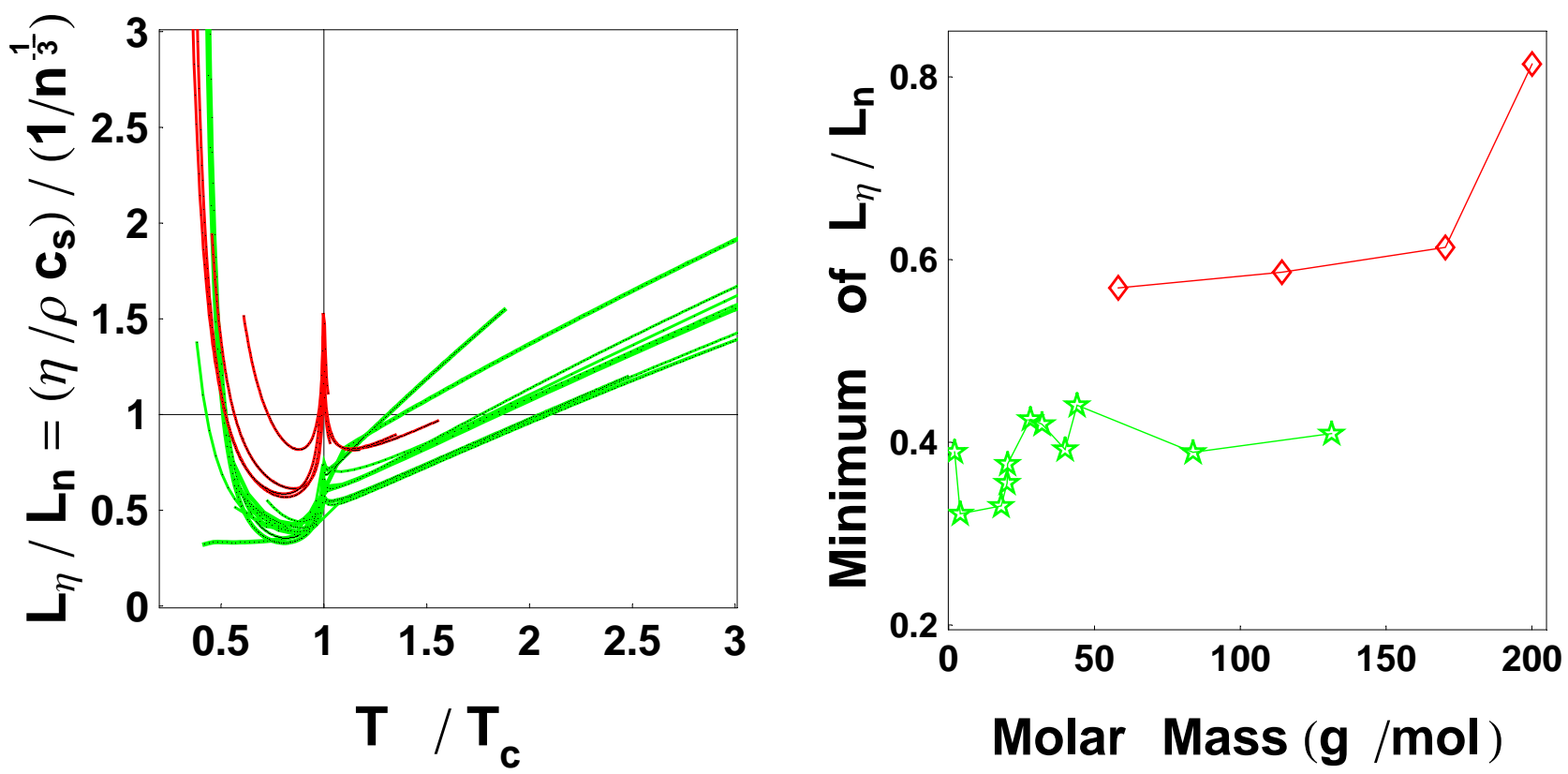

FIG. 1: (Color online) (left panel) Fluidity measure $\mathcal{F}=L_{\eta} / L_{n}$ versus $T / T_{c}$ for fifteen different substances at fixed critical pressure $P=P_{c}$, see text for more details. The sharp peaks centered at $T_{c}$ are due to the actual divergence of the shear viscosity at the critical point. (right panel) The minimum of the fluidity measure $\mathcal{F}$ for various substances (as obtained from respective curves in the left panel) versus their molar masses, with the stars (from left to right) for $\mathrm{H}_{2},{ }^{4} \mathrm{He}, \mathrm{H}_{2} \mathrm{O}, \mathrm{D}_{2} \mathrm{O}, \mathrm{Ne}$, $\mathrm{N}_{2}, \mathrm{O}_{2}, \mathrm{Ar}, \mathrm{CO}_{2}, \mathrm{Kr}, \mathrm{Xe}$, and the diamonds(from left to right) for $\mathrm{C}_{4} \mathrm{H}_{10}, \mathrm{C}_{8} \mathrm{H}_{18}, \mathrm{C}_{12} \mathrm{H}_{26}, \mathrm{C}_{4} F_{8}$.

their respective critical pressure $P_{c}$, including: Hydrogen $\left(\mathrm{H}_{2}\right)$, Helium-4 $\left({ }^{4} \mathrm{He}\right)$, Water $\left(\mathrm{H}_{2} \mathrm{O}\right)$, Deuterium oxide $\left(\mathrm{D}_{2} \mathrm{O}\right)$, Neon $(\mathrm{Ne})$, Nitrogen $\left(\mathrm{N}_{2}\right)$, Oxygen $\left(\mathrm{O}_{2}\right)$, Argon $(\mathrm{Ar})$, Carbon Dioxide $\left(\mathrm{CO}_{2}\right)$, Krypton $(\mathrm{Kr})$, Xenon $(\mathrm{Xe})$, Isobutane $\left(\mathrm{C}_{4} \mathrm{H}_{10}\right)$, Octane $\left(\mathrm{C}_{8} \mathrm{H}_{18}\right)$, Dodecane $\left(C_{12} H_{26}\right)$, Octafluorocyclobutane $\left(C_{4} F_{8}\right)$. These substances cover a wide range of molar mass, chemical structure and complexity, with their respective critical temperature $T_{c}$ and pressure $P_{c}$ differing by orders of magnitude. Despite such huge differences, their fluidity curves resemble each other not only in shape but even quantitatively. In particular in their good liquid regime roughly the "valley" region at $\sim 0.7-1 T c-$ they all show amazingly similar fluidity. To further expose the similarity, in the right panel of Fig 1 we show the value of the fluidity measure $\mathcal{F}$ at its minimum versus the substances' molecular molar masses. From this plot, roughly two bands can be identified: the green stars spread in a narrow band of $\mathcal{F} \in(0.3,0.45)$ while spanning two-orders-of magnitude in molar mass which include all 11 non-organic substances; the red diamonds with roughly twice bigger $\mathcal{F}$, include 4 organic substances with much more complicated molecular structures (e.g. chains) which, not surprisingly, lead to more dissipation. Even so, the splitting in fluidity between the two bands is merely a $\hat{O}(1)$ factor rather than any order-of-magnitude difference. Nevertheless as one can imagine, with increasing chemical complexity and molecular mass, the fluidity of more complex systems like e.g. engine oil may deviate significantly from what are shown in the figure.
To conclude the study of critical fluids, there appears to be certain universality of the newly proposed fluidity measure $\mathcal{F}$, indicating that "a good fluid is a good fluid" despite many other details regarding the microscopic degrees of freedom.

\section{B. Supercritical Fluids}

While much attention has been paid to critical fluids, the fluidity of a fluid with significantly larger pressure $P>>P_{c}$ has been little discussed in the context of heavy ion collisions and QCD matter: so let us next explore this region. In the literature, this region is often referred to as the "supercritical fluid" region [21], defined on a typical substance's $T-P$ phase diagram (with critical point $\left.T_{c}, P_{c}\right)$ as:

$$
\text { supercritical : } \quad T>T_{c} \& P>P_{c}
$$

In particular we want to explore the deeply supercritical region with $P>>P_{c}$. Taking water as an example, in Fig.2(left) we plot the fluidity measure $\mathcal{F}$ versus $T / T c$ for fifteen different fixed pressure values ranging from $5 \mathrm{MPa}$ all the way to $1000 \mathrm{MPa}$ (note for water $P_{c}=22 \mathrm{MPa}$ ). The dashed (green) curve is for fixed critical pressure $P=P_{c}=22 \mathrm{MPa}$, while the four solid (red) curves above it are for $P<P_{c}$, and the ten solid (blue) curves below it are for $P>P_{c}$. As can be seen from the plot, the curves change shape gradually and the fluidity becomes better and better with increasing $P$. The "valley" 

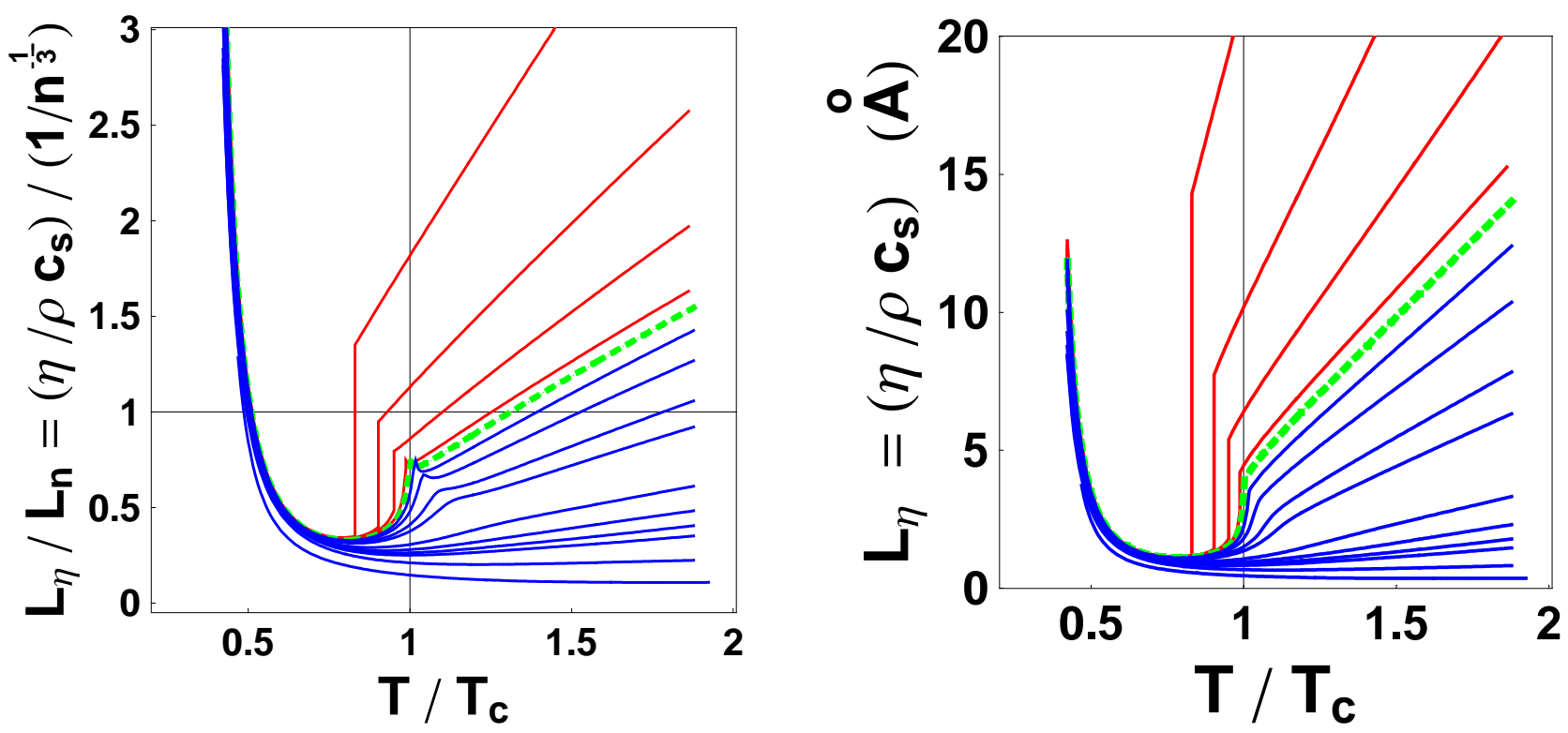

FIG. 2: (Color online) The dimensionless fluidity measure $\mathcal{F}$ (left panel) and the length scale, $L_{\eta}$ (in units of $\AA$ ) (right panel) versus $T / T_{c}$ for water at fifteen different fixed pressure values. In both panels, the dashed (green) curve is for fixed critical pressure $P=P_{c}=22 \mathrm{MPa}$, while the four solid (red) curves above it are for $P<P_{c}$, and the ten solid (blue) curves below it are for $P>P_{c}$, with the respective pressure values for each curve (from top down) being $P=5,10,15,20,22,25,30,40,50,100,150,200,250,500,1000 \mathrm{MPa}$.

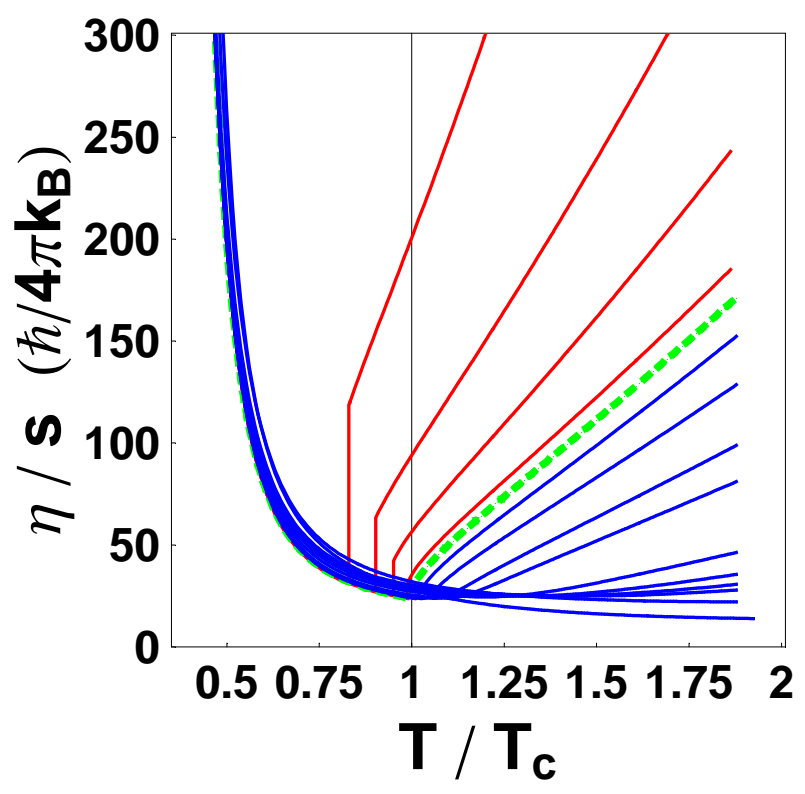

FIG. 3: (Color online) The shear-viscosity-entropy-densityratio $\eta / s$ (in unit $\hbar /\left(4 \pi k_{B}\right)$ ) versus $T / T c$ for water at fifteen different fixed pressure values. The dashed (green) curve is for fixed critical pressure $P=P_{c}=22 \mathrm{MPa}$, while the four solid (red) curves above it are for $P<P_{c}$, and the ten solid (blue) curves below it are for $P>P_{c}$, with the respective pressure values for each curve (from top down) being $P=$ $5,10,15,20,22,25,30,40,50,100,150,200,250,500,1000 \mathrm{MPa}$. where $\mathcal{F}$ remains small and relatively flat becomes much wider and eventually flattens out substantially above $T_{c}$ for $P>>P c$. To quantify the remarkable fluidity of the supercritical fluid, we note that the minimum on the $P=P_{c}$ curve has a value for the fluidity $\mathcal{F}_{\min }(P c) \approx 0.33$ while the minimum for the $P=1000 \mathrm{MPa} \approx 45 P_{c}$ curve is at $\mathcal{F}_{\min }(45 P c) \approx 0.11$, getting smaller by a factor of 3 and remaining close to the minimum within a rather broad temperature region!

It is also interesting to examine whether the length scale $L_{\eta}$ itself shows similar trends, as $L_{\eta}$ is the essential scale for discussing the applicability of hydrodynamics where it is to be compared with the external scale characterizing the variation of flow field. In the right panel of Fig,2 we plot $L_{\eta}$ itself for the same conditions and find that, similar to the fluidity measure $\mathcal{F}$, the scale $L_{\eta}$ also becomes considerably smaller in supercritical water.

It should be mentioned that the same observation is also true for other fluids that we examined, like Helium-4, Nitrogen, etc. Furthermore such behavior is not specific to our fluidity measure. In Fig 3 we plot the widely used ratio of shear viscosity over entropy-density, $\eta / s$, which show the same qualitative behavior.

The main lessons, as we emphasize again, are (a) for a given substance, the best fluidity is not necessarily achieved close to the critical point and (b) when going deeper into the supercritical regime its fluidity becomes much better. 


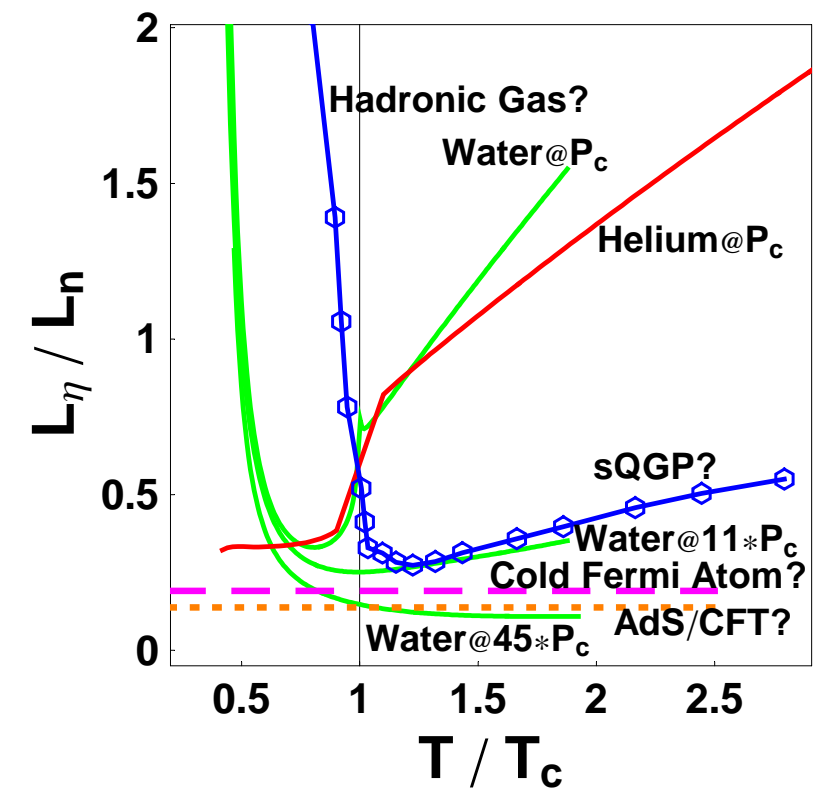

FIG. 4: (Color online) Comparison of fluidity measure for various fluids (see text for more details). The curves with question marks indicate current estimates of the respective fluidity with possible uncertainty, while the curves for Helium at $P_{c}$ and for water at $P_{c}, 11 P_{c}, 45 P_{c}$ are from actual data.

\section{Comparison of Various Fluids}

Finally we attempt to compare various fluids of great current interests in terms of the new fluidity measure $\mathcal{F}$ we have proposed and studied above. The results are shown in Fig 4. Below we give the details of how the curves for QCD, Cold Fermi Atom, and AdS/CFT are obtained.

For the QCD system, we have used a parametrization of the viscosity $\eta$ by Hirano and Gyulassy in [24]: one uses $\eta / T_{c}^{3} \approx T / T_{c}$ for hadronic gas (H.G.) below $T_{c}$, and $\eta / T_{c}^{3} \approx\left(T / T_{c}\right)^{3}\left[1+\mathcal{W}(T) \ln \left(T / T_{c}\right)\right]^{2}$ for the quark-gluon plasma(sQGP) in the temperature interval $T / T_{c} \in[1-3]$ with

$[\mathcal{W}(T) /(4 \pi)]=\left(9 \beta_{0}^{2}\right) \cdot\left[80 \pi^{2} K_{S B} \ln \left(4 \pi / g^{2}(T)\right)\right]^{-1}$ interpolating to pQCD results for $T>>T_{c}$, where the parameters are given as $\beta_{0}=10, K_{S B}=12$ and the running coupling

$\left[g^{2}(T)\right]^{-1}=\left(9 / 8 \pi^{2}\right) \ln (2 \pi T / \Lambda) \quad+$ $\left(4 / 9 \pi^{2}\right) \ln (2 \ln (2 \pi T / \Lambda)) \quad($ with $\Lambda \approx 190 M e V)$ ( see [24] [11] for more details). The enthalpy density $w=\epsilon+p$ and speed of sound $c_{s}$ are taken from recent lattice results by Karsch et al [25] for $2+1$ flavor QCD with $m_{\pi} \approx 220 \mathrm{MeV}$. As we mentioned before, $L_{n}$ is estimated by $1 /\left(s / 4 k_{B}\right)^{1 / 3}$ with the entropy density also taken from [25].

For the strongly coupled AdS/CFT system, the shear viscosity is well known to be $\eta / s=1 /(4 \pi)[9]$. As we also pointed out before, there is a short-range order at the length scale $L_{n} \sim 1 / T$ however the pre-factor is not accurately determined. We simply use $L_{n}=1 / T$ as an estimate. This gives the fluidity $\mathcal{F}=\sqrt{3} /(4 \pi) \approx 0.138$.

For the Cold Fermi Atom gas, its shear viscosity has been measured near its Feshbach resonance by Thomas et al in [26] for a certain range of system energy by analyzing the damping of collective modes in the atomic cloud (see also related work in [27]). As a benchmark, we take the lowest viscosity found from the measurement (see Fig.4 in [26]) which is $\eta \approx 0.214 \hbar n$. The speed of sound has also been measured by the same group in [28], from which we take the value $c_{s} \approx 0.3632 v_{F}$ near the Feshbach resonance. Since the Fermi velocity $v_{F}=\hbar k_{F} / m$, the mass density $\rho=m n$, and the Fermi momentum $k_{F} / n^{1 / 3}=\left(3 \pi^{2}\right)^{1 / 3}$, we obtain for the fluidity measure $\mathcal{F} \approx 0.214 /\left(0.3632 \cdot\left(3 \pi^{2}\right)^{1 / 3}\right) \approx 0.191$.

The question marks in Fig 4 indicate that the current estimates presented above may carry sizable uncertainties, and we expect the knowledge on these systems will become more accurate with time.

We finally come to the comparison in Fig 4, As one can see, the critical fluids (water and Helium-4 at $P_{c}$ ) have a somewhat worse fluidity than the QCD, AdS/CFT and Cold Fermi Atom systems. Supercritical water at $P=11 P c$, on the other hand, already has a fluidity comparable to the QGP while at $P=45 P c$ the fluidity of supercritical water appears to be better than that of the recently discussed "nearly perfect fluids".

\section{A SUPERCRITICAL QCD FLUID AT RHIC?}

The fluidity study in the previous section, in particular on the supercritical fluid, naturally leads to the following interesting question: Are we observing a supercritical QCD fluid at RHIC?

As is well known, what is usual referred to as $T_{c} \approx$ $170 \sim 190 \mathrm{MeV}$ in QCD is not a true second-order phase transition temperature but rather the temperature for a rapid crossover at $\mu=0$ [25]. There is, however, a (hypothetical) Critical-End-Point (CEP) at $\left(T_{C E P}, \mu_{C E P}\right)$ on the QCD phase diagram marking the end of a plausible first-order phase transition at low $T$ but high $\mu$ (see e.g. [29] and references therein). Accurate determination of the CEP from lattice QCD [30 34] and unambiguous observation of the CEP from heavy ion collisions [12, 35, 36] are among the most interesting and exciting goals of QCD research. While currently the position of the CEP is not well constrained, it is expected to have lower temperature than that of the QCD crossover transition at vanishing baryon density, $T_{C E P}<T_{c}$. Being very aware on the present uncertainty of the actual location of the QCD critical end point, let us, solely for demonstration purposes and for the sake of the argument, assume that its location is close to the estimate of ref. 32], which suggests that $T_{C E P} \approx 0.94 T_{c}, \mu_{C E P} \approx 1.8 T_{C E P}$. This location is indicated in Fig 5 as the filled black circle along with a question mark.

Next if we adopt the definition of supercriticality as in 


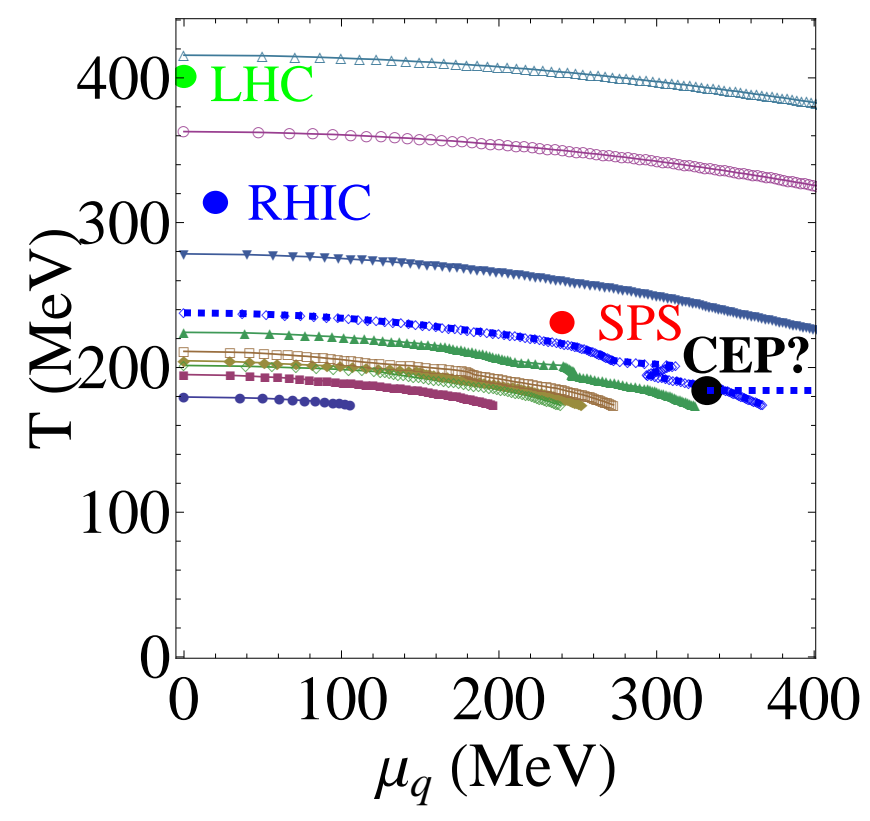

FIG. 5: (Color online) Schematic isobaric contours (i.e. with constant pressure along each line) on the QCD $T-\mu$ phase diagram with the filled black circle indicating a possible position of the hypothetical Critical-End-Point (CEP); the dashed blue horizontal short line stretching from the CEP to the right is included to indicate the $T=T_{C E P}$ boundary (see text for more details). The filled red, blue and green circles indicate estimates of the initial $(T, \mu)$ reachable at SPS, RHIC and LHC, respectively.

Eq.(25) for QCD, we need to know the constant-pressure lines on the QCD phase diagram typically plotted in terms of $T-\mu$. To schematically construct these lines, we make use of the Taylor expansion of the pressure $P(T, \mu)$ with respect to $\mu$ :

$$
\begin{aligned}
P(T, \mu)=T^{4} \times \quad & {\left[P_{0}(T)+\frac{1}{2} \chi_{2}^{B}(T)\left(\frac{\mu}{T}\right)^{2}\right.} \\
& \left.+\frac{1}{24} \chi_{4}^{B}(T)\left(\frac{\mu}{T}\right)^{4}+\ldots\right]
\end{aligned}
$$

with $\left.\chi_{2 n}^{B} \equiv \frac{\partial^{2 n}\left(P / T^{4}\right)}{\partial(\mu / T)^{2 n}}\right|_{\mu=0}$ the baryonic susceptibilities 32, 33, 37, 38, 40]. In order to construct the equalpressure lines shown in Fig 5 we have used the lattice QCD results from Cheng et al for $P_{0}(T)[25]$ and $\chi_{2}^{B}, \chi_{4}^{B}$ 37].

The blue curve in Fig 5 going right through the indicated CEP point indicates the constant-pressure line with fixed critical pressure $P=P(C E P)$ which, together with the $T=T_{C E P}$ line (dashed blue horizontal one stretching from the CEP to the right) form the boundary above which there is the $Q C D$ supercritical region. We emphasize that above the boundary the pressure $P \sim T^{4}$ increases very rapidly. As a result the QGP quickly enters deeply into the supercritical regime where $P \gg P(C E P)$. To give an idea: the pressures of the lines in units of the critical pressure $P(C E P)$ (value on the blue curve) are (from bottom to top) $0.05,0.08,0.13,0.23,0.27,0.35$, $0.63,1,2.7,10,18$, respectively. Of course with improved lattice results for pressure, CEP and (higher order) susceptibilities a more accurate equal-pressure map for QCD matter will become available.

We now discuss a few implications for heavy ion collisions experiments. As a precaution, the following discussions are by no means intended to provide quantitative statements but rather qualitative yet interesting ideas.

(I) At current RHIC energy $(\sqrt{s}=200 \mathrm{AGeV})$, Fig 5 indicates that most of the QGP phase of the created matter is likely in the supercritical region. This may very well be the reason why such good fluidity has been observed. If a relationship between good fluidity and supercriticality is indeed true for the QCD matter just as it is for water, then there will be a sensitive dependence of the fluidity on the actual position of the CEP. For example, the matter created at RHIC might have most of its evolution being in the supercritical region in case $T_{C E P}$ and $P_{C E P}$ are considerably lower than indicated in the Fig 5 . On the other hand, the matter created at SPS $(\sqrt{s} \simeq 20 \mathrm{GeV})$ seems to have its initial pressure and temperature already rather close to the critical one which would result in poor fluidity. In principle these regions (supercritical, near-CEP, below-CEP) of very different fluidity can be accessed experimentally by tuning the center of mass energy $\sqrt{s}$ which changes the initial densities (see e.g. [41]) as well as the freeze-out points (see e.g. [42] 43]) in such collisions. To which extend this difference in fluidity transforms into measurable effects, such as non-hydrodynamic behavior and thus less elliptic flow, is a non-trivial question. As we will discuss in the next section, besides the fluidity the actual size of the system is essential for the applicability of hydrodynamics. Our estimates (see Fig [6) show that even for the very good fluidity assumed for a strongly interacting QGP, denoted as "sQGP" in Fig 4 a hydrodynamic description for the fireball expansion is a best marginal at SPS energies. The reason is that, according to our estimate, the system starts close to the phase transition and, thus, reaches the hadronic phase while still comparatively small in size. Consequently, it may be difficult to tell if the system enters a regime of reduced fluidity from flow studies alone. Of course if our estimates are wrong and one finds evidence that the initial entropy density reached is considerably larger, a change in the fluidity may show up in the systematics of elliptic flow measurements, such as its dependence on beam energy and system size.

(II) Based on the possibility of a mostly supercritical QCD matter to be created in experiments at the Large Hadron Collider (LHC) (see Fig [5), one is led to predict an even better fluidity to be observed at LHC than at RHIC. And since for the same fluidity the conditions for hydrodynamics are more favorable for LHC energies than for RHIC, we would expect nearly ideal hydrodynamic evolution at LHC. 


\section{APPLICABILITY OF HYDRODYNAMICS IN HEAVY ION COLLISIONS}

In this section we turn to a discussion of applicability of hydrodynamics in heavy ion collisions, particularly for SPS, RHIC and LHC. As already discussed in the introduction, the fluidity of the underlying matter is not the only determining factor: the "best" (in terms of fluidity) fluid may cease to flow if put into a sufficiently narrow pipe. In other words, the applicability of hydrodynamics depends equally on both the internal properties of the substance, i.e. its fluidity $\mathcal{F}$ or $L_{\eta}$, and the external settings, i.e the typical length scale $L_{s}$ of the variation of the flow field in the system under consideration ${ }^{6}$ : one useful criteria, equivalent to the famous Knudsen number, is a ratio of the two $L_{\eta} / L_{s}$.

In order to derive a quantitative criterion, we again use the example of sound dispersion discussed before. By requiring that a propagating sound wave has to complete at least one full period before its amplitude damps by a factor $e^{-1}$ due to the imaginary part, we arrive at a minimal wave length $\lambda_{\min }=\frac{8 \pi^{2}}{3} L_{\eta}$. For a sound wave the length scale characterizing the flow field variations simply the wavelength, $L_{s} \approx \lambda$. Thus we arrive at the following criterion for the applicability of hydrodynamics:

$$
\frac{L_{\eta}}{L_{s}}<\frac{3}{8 \pi^{2}} \approx 0.038
$$

The length $L_{\eta}(T)$ for QCD (at $\mu=0$ ), shown in Fig 6(left), is determined as described section [IIC. It relies on a parametrization for $\eta(T)$ from the HiranoGyulassy [24], and we note that the actual shear viscosity of QCD matter, once it is known, may be quite different. The temperature dependence of $L_{\eta}$ shown in the plot features a steep drop from the hadronic phase to about $T \simeq 1.1 T_{c}$, followed by a rather constant value for temperatures above, $T \gtrsim 1.1 T_{c}$. One may expect $L_{\eta}$ becomes large again at very high temperatures $T>>T_{c}$ where a weakly coupled QGP describable by pQCD takes over. In principle $L_{\eta}$ also depends on the baryon-number chemical potential, $\mu_{B}$, and this dependence shall be taken into account when discussing collisions at low energy (such as SPS) where $\mu_{B}$ could be sizable. However currently very little is known about such dependence, and we here use the zero $\mu$ result also for SPS.

We next give a rough estimate for the typical length scale $L_{s}$ of flow field variance for heavy ion collisions at different $\sqrt{s}$. At early times, right after the collision,

\footnotetext{
6 We re-emphasize that the length scale $L_{\eta}$ is a well defined quantity depending only on macroscopic variables that are measurable and/or calculable. Furthermore unlike the mean-free-path which depends on quasi-particle picture (see e.g. discussions in [15]), the length scale $L_{\eta}$ remains a useful and meaningful scale even for strongly coupled systems.

7 In this discussion we focus only on central collisions which make
}

the system size is limited mainly by its longitudinal extent and the scale governing the variation in the fluid can be estimated to be about $2 \tau$. At late time when $\tau$ becomes larger than the nuclear radius $\sim R_{A}$ the limiting scale is set by the transverse size of the fireball which is slightly larger than $R_{A}$ and slowly grows. For the purpose of our rough estimate, we adopt a simple prescription: $L_{s} \approx 2 \tau$ for $\tau<R$ and $L_{s} \approx 2 R$ for $\tau \geq R$ with $R=8 \mathrm{fm}$ for $\mathrm{AuAu}$ and $\mathrm{PbPb}$ collisions. For the collision dynamics which determines the temperature evolution $s(\tau)$ (or inversely $\tau(s)$ ), we simply use the Bjorken flow relation $s(\tau)=s_{0}\left(\tau_{0}\right) \cdot \tau_{0} / \tau$, which up to $\tau \sim R$ is a reasonable approximation. For the initial condition we follow estimates used in hydrodynamic modelling (see e.g. [41]) and assume equilibration at $\tau_{0}=1 \mathrm{fm}$, with fireball center entropy densities (in central collisions) to be $s_{0}=24 \mathrm{fm}^{-3}$ at SPS, $s_{0}=70 \mathrm{fm}^{-3}$ at RHIC, and $s_{0}=154 \mathrm{fm}^{-3}$ at LHC.

Finally we turn to the discussion of the applicability of hydrodynamics given the criteria of Eq[27. The ratio $L_{\eta} / L_{s}$ in units of $3 /\left(8 \pi^{2}\right)$ is plotted in Fig [6)(right) for SPS(red diamonds), RHIC(blue stars), and LHC(green boxes). The thick dashed horizontal line indicates the equality of the condition expressed by Eq27. If the system is considerably below this line a hydrodynamic description should be a reasonable approximation. If it finds itself considerably above corrections to hydrodynamics from higher orders in derivatives or an even nonhydrodynamic description are called for. To quantify the previous statement, if the system finds itself at a value of $\left(L_{\eta} / L_{s}\right) /\left(3 / 8 \pi^{2}\right)=2$, such as the points for SPS energies on Fig.6(left), then the amplitude of a sound mode after propagating the distance of one wavelength will be reduced by a factor of $e^{2} \simeq 7$, and for a value of $\left(L_{\eta} / L_{s}\right) /\left(3 / 8 \pi^{2}\right)=5$ the reduction would be $e^{5} \simeq 150$ ! Given our estimate and the assumptions which it is based on, a hydrodynamic description is not a good approximation for SPS, whereas for RHIC and even more so for LHC energies, it seems to be more or less justified ${ }^{8}$. Such differences from SPS to RHIC and LHC lie in the different time evolution due to the different initial densities: when cooling down into the region where $L_{\eta}$ starts rising abruptly near and below $T_{c}$, the SPS fireball still has a rather small size (longitudinally) while the RHIC and LHC fireballs already becomes large with a size about $2 R$ and hence have their hydrodynamic evolution extended much longer. While our estimates contain uncertainties and should by no means considered to be precise, we have

the estimation easier. Generally speaking when going from central to peripheral collisions one expects hydrodynamics to be less and less applicable.

8 We note that even for RHIC and LHC the first fm or so of the evolution is above the "criteria line" due to the rather small system size. Whether this has a profound effect (such as in entropy generation) or not is unclear at this time (see a very interesting discussion in [44]). 

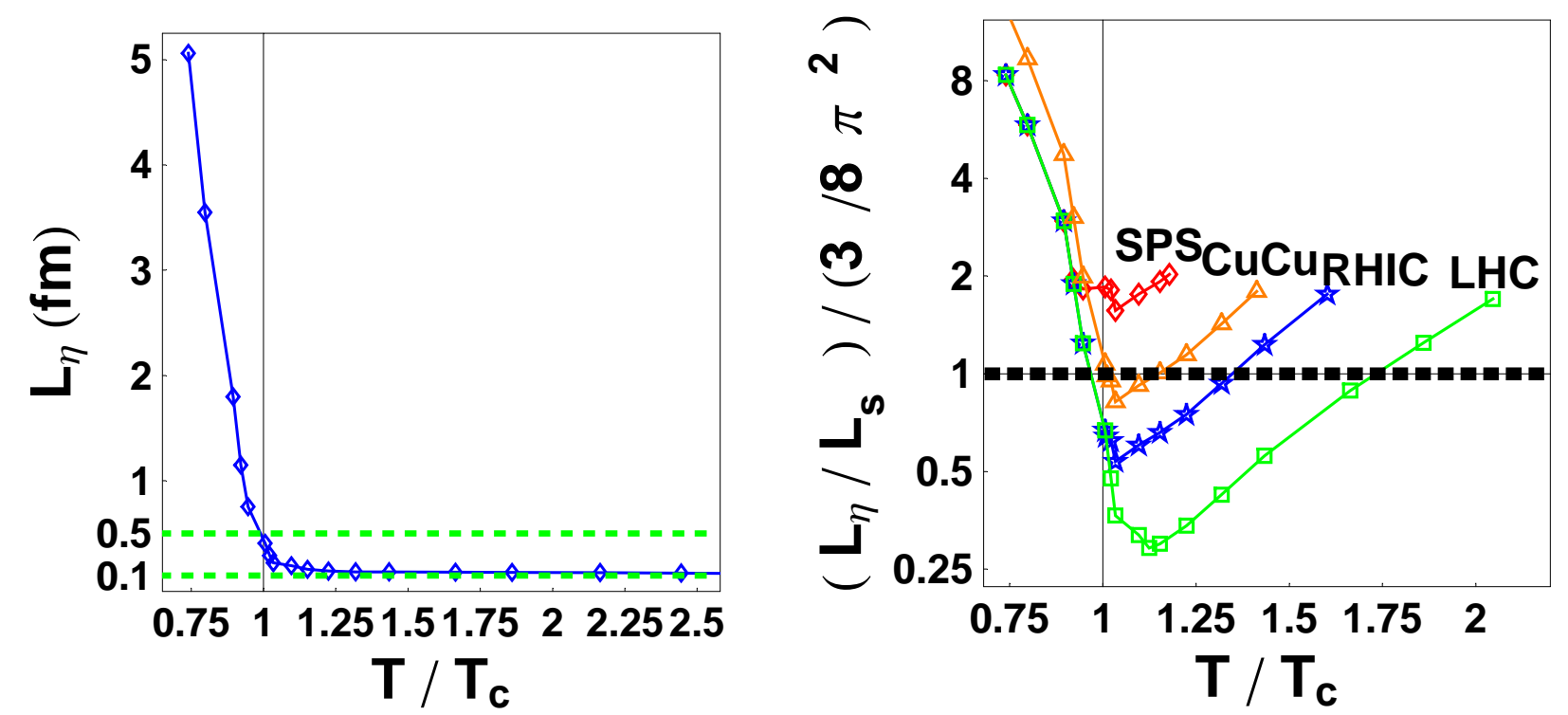

FIG. 6: (Color online) (left panel) The length scale $L_{\eta}=\frac{\eta}{\left(w / c^{2}\right) c_{s}}$ (in unit fm) versus $T / T_{c}$ for QCD at $\mu=0$, with $\eta$ from parametrization in 24] and $w, c_{s}$ from lattice data 25]. The dashed horizontal lines at $0.1 \mathrm{fm}$ and $0.5 \mathrm{fm}$ are included to guide the eyes. (right panel) The criteria (27) for applicability of hydrodynamics versus $T / T_{c}$ at SPS(red diamonds), RHIC(blue stars), and LHC(green boxes), with the thick dashed horizontal line indicating the borderline below which hydrodynamics may be a good description according the criteria (see text for more details). A line for $\mathrm{CuCu}$ (orange triangles) collisions at RHIC energy $(200 \mathrm{AGeV})$ is also presented.

provided a semi-quantitative picture for evaluating the applicability of hydrodynamics with varying $\sqrt{s}$, which implies at LHC the fireball expansion shall be even better described by hydrodynamics as compared to RHIC.

Besides the beam energy $\sqrt{s}$, a change of the system size should also affect the conditions for the applicability of hydrodynamics. For example, RHIC has done experiments with both $\mathrm{AuAu}$ collisions and $\mathrm{CuCu}$ collisions, both at full energy, and it would be interesting to examine and compare the applicability of hydrodynamics for these two different systems. In Fig 6 (right) we have included a calculation of the same applicability measure for $\mathrm{CuCu}$ (orange triangle) collisions at $200 \mathrm{AGeV}$, with a initial center entropy density $s_{0}=45 \mathrm{fm}^{-3}$ and a transverse size parameter $R=5 \mathrm{fm}$. The curve indicates that hydrodynamics is marginally applicable for such collisions, as both the initial density and the transverse size of the matter formed in $\mathrm{CuCu}$ collisions are smaller that those in $\mathrm{AuAu}$ collisions at the same energy.

\section{SUMMARY}

In summary, we have discussed the fluidity of the hot and dense QCD matter as produced in ultrarelativistic heavy ion collisions in comparison with various other, well known fluids. In particular we have suggested its possible supercriticality based on insights gained from studying more conventional fluids like water. We have discussed several aspects relevant for a proper comparison of non-relativistic and relativistic fluids, both from thermodynamics and hydrodynamics perspectives. A new fluidity measure $\mathcal{F}=L_{\eta} / L_{n}$ is then proposed, which shows certain universality in the good fluid regime for a remarkable diverse set of critical fluids. We have further demonstrated that the fluidity is enhanced in the supercritical fluid regime on a fluid's phase diagram. These studies inspired us to conjecture that the seemingly good fluidity of the QCD matter at RHIC may actually be related to its supercriticality with respect to the CriticalEnd-Point on the QCD phase diagram. This observation, if true, has far-reaching consequences for heavy ion collisions experiments: (a) the loss of such good fluidity at certain lower beam energy which is sensitively related to the position of the long sought CEP; (b) an even better fluidity may be expected at higher beam energy, which will soon be tested by the LHC heavy ion program. Finally we have analyzed the applicability of hydrodynamics for the fireball evolution in heavy ion collisions at various energies. Our analysis was based on a model parametrization for the shear-viscosity and a rough estimate of the relevant length scales governing the fireball expansion. Given these assumptions we find that hydrodynamics should be applicable for collisions at RHIC and LHC energies but not for SPS energies. 


\section{Acknowledgments}

The authors are grateful to Ulrich Heinz, Roy Lacey, Dirk Rischke, Thomas Schaefer, Edward Shuryak, and $\mathrm{Nu} \mathrm{Xu}$ for valuable communications. The work is sup- ported by the Director, Office of Energy Research, Office of High Energy and Nuclear Physics, Divisions of $\mathrm{Nu}-$ clear Physics, of the U.S. Department of Energy under Contract No. DE-AC02-05CH11231.
[1] S. A. Voloshin, A. M. Poskanzer and R. Snellings, arXiv:0809.2949 [nucl-ex].

[2] U. W. Heinz, arXiv:0901.4355 [nucl-th]. D. A. Teaney, arXiv:0905.2433 [nucl-th]. P. Romatschke, arXiv:0902.3663 [hep-ph].

[3] T. Schaefer and D. Teaney, Rept. Prog. Phys. 72, 126001 (2009).

[4] J. Liao and E. Shuryak, Phys. Rev. C 75, 054907 (2007); Phys. Rev. Lett. 101, 162302 (2008); Phys. Rev. Lett. 102, 202302 (2009). M. N. Chernodub and V. I. Zakharov, Phys. Rev. Lett. 98, 082002 (2007).

[5] Z. Xu, C. Greiner and H. Stocker, Phys. Rev. Lett. 101, 082302 (2008). J. Noronha-Hostler, J. Noronha and C. Greiner, Phys. Rev. Lett. 103, 172302 (2009).

[6] R. D. Pisarski, Phys. Rev. D 74, 121703 (2006). Y. Hidaka and R. D. Pisarski, Phys. Rev. D 78, 071501 (2008).

[7] J. Liao and V. Koch, Phys. Rev. Lett. 103, 042302 (2009).

[8] V. Koch, arXiv:0908.3176 [nucl-th].

[9] G. Policastro, D. T. Son and A. O. Starinets, Phys. Rev. Lett. 87, 081601 (2001); P. Kovtun, D. T. Son and A. O. Starinets, Phys. Rev. Lett. 94, 111601 (2005).

[10] T. D. Cohen, Phys. Rev. Lett. 99, 021602 (2007). A. Cherman, T. D. Cohen and P. M. Hohler, JHEP 0802, 026 (2008).

[11] L. P. Csernai, J. I. Kapusta and L. D. McLerran, Phys. Rev. Lett. 97, 152303 (2006).

[12] R. A. Lacey et al., Phys. Rev. Lett. 98, 092301 (2007).

[13] A. Bonasera and L. P. Csernai, Phys. Rev. Lett. 59, 630 (1987); A. Bonasera, L.P. Csernai and B. Schurmann, Nucl. Phys. A 476, 159 (1988).

[14] C. Gombeaud, T. Lappi and J. Y. Ollitrault, Phys. Rev. C 79, 054914 (2009). I. Bouras et al., Phys. Rev. Lett. 103, 032301 (2009).

[15] B. Betz, D. Henkel and D. H. Rischke, Prog. Part. Nucl. Phys. 62, 556 (2009).

[16] K. Huang, "Statistical Mechanics", 2nd ed., Wiley, 1987.

[17] L. D. Landau and E. M. Lifshitz, "Fluid Mechanics", 2nd ed., Butterworth-Heinemann, 1987.

[18] S. Weinberg, "Gravitation and Cosmology: Principles and Applications of the General Theory of Relativity", John Wiley \& Sons, 1972.

[19] P. Danielewicz and M. Gyulassy, Phys. Rev. D 31, 53 (1985).

[20] T. Schaefer, arXiv:0906.5399 [physics.flu-dyn].

[21] E. Ulrich Franck, J. Chem. Thermodynamics 19, 225 (1987); F. Bencivenga et al, Europhys. Lett. 75, 70 (2006); F. Gorelli et al, Phys. Rev. Lett. 97, 245702
(2006); V. S. Nikolayev et al, Phys. Rev. E 67, 061202 (2003).

[22] H. B. Meyer, Phys. Rev. D 79, 011502 (2009).

[23] National Institute of Standards and Technology (NIST) Standard Reference Database No. 69, June 2005, NIST Chemistry WebBook: http://webbook.nist.gov/chemistry/. See, in particular, Thermophysical Properties of Fluid Systems: High Accuracy Data for a Select Group of Fluids.

[24] T. Hirano and M. Gyulassy, Nucl. Phys. A 769, 71 (2006).

[25] M. Cheng et al., Phys. Rev. D 77, 014511 (2008).

[26] A. Turlapov, et al, J. Low. Temp. Phys. 150, 567 (2008).

[27] T. Schaefer, Phys. Rev. A 76, 063618 (2007).

[28] J. Joseph, et al, Phys. Rev. Lett. 98, 170401 (2007).

[29] M. A. Stephanov, PoS LAT2006, 024 (2006).

[30] Z. Fodor and S. D. Katz, JHEP 0203, 014 (2002); JHEP 0404, 050 (2004).

[31] P. de Forcrand and O. Philipsen, Nucl. Phys. B 673, 170 (2003).

[32] R. V. Gavai and S. Gupta, Phys. Rev. D 71, 114014 (2005); Phys. Rev. D 72, 054006 (2005).

[33] C. R. Allton et al., Phys. Rev. D 71, 054508 (2005).

[34] O. Philipsen, PoS LAT2005, 016 (2006) [PoS JHW2005, 012 (2006)].

[35] M. A. Stephanov, K. Rajagopal and E. V. Shuryak, Phys. Rev. Lett. 81, 4816 (1998); Phys. Rev. D 60, 114028 (1999).

[36] R. A. Lacey, N. N. Ajitanand, J. M. Alexander, P. Chung, J. Jia, A. Taranenko and P. Danielewicz, arXiv:0708.3512 [nucl-ex].

[37] M. Cheng et al., Phys. Rev. D 79, 074505 (2009).

[38] J. Liao and E. V. Shuryak, Phys. Rev. D 73, 014509 (2006); Nucl. Phys. A 775, 224 (2006).

[39] K. y. Kim and J. Liao, Nucl. Phys. B 822, 201 (2009).

[40] V. Koch, arXiv:0810.2520 [nucl-th].

[41] G. Kestin and U. W. Heinz, Eur. Phys. J. C 61, 545 (2009).

[42] P. Braun-Munzinger, D. Magestro, K. Redlich and J. Stachel, Phys. Lett. B 518, 41 (2001). P. Braun-Munzinger, K. Redlich and J. Stachel, arXiv:nucl-th/0304013

[43] J. Randrup, Phys. Rev. C 79, 054911 (2009). J. Randrup and J. Cleymans, arXiv:0905.2824 [nucl-th].

[44] M. Lublinsky and E. Shuryak, Phys. Rev. C 76, 021901 (2007); Phys. Rev. D 80, 065026 (2009). 